\title{
Article \\ Targeting Tat-TAR RNA Interaction for HIV-1 Inhibition
}

\author{
Awadh Alanazi, ${ }^{1}$ Andrey Ivanov, ${ }^{2}$ Namita Kumari, ${ }^{1}$ Xionghao Lin, ${ }^{2,4}$ Songping Wang, ${ }^{1}$ Dmytro \\ Kovalskyy, ${ }^{5}$ and Sergei Nekhai ${ }^{1,2,3, *}$ \\ 1 Department of Microbiology, College of Medicine, Howard University, Washington DC, 20059, \\ USA; awadhaalanazi@gmail.com \\ 2 Center for Sickle Cell Disease, College of Medicine, Howard University, Washington DC, 20059, \\ USA; andrey.ivanov@howard.edu; namita.kumari@howard.edu; xionghao.lin@howard.edu; \\ swang.howard.edu; \\ 3 Department of Medicine, College of Medicine, Howard University, Washington DC, 20059, USA \\ 4 Department of Oral and Maxillofacial Pathology, College of Dentistry, Howard University, \\ Washington DC, 20059, USA \\ 5 Department of Biochemistry, University of Texas Health Science Center, San Antonio, TX 78229, \\ USA; d.kovalskyy@gmail.com>
}

* Correspondence: snekhai@howard.edu (S.N.)

\begin{abstract}
HIV-1 Tat protein interacts with TAR RNA and recruits CDK9/cyclin T1 and other host factors to induce HIV-1 transcription. Thus Tat-TAR RNA interaction, which is unique for HIV-1, represents an attractive target for anti-HIV-1 therapeutics. To target Tat-TAR RNA interaction, we used a crystal structure of TAR RNA with acetylpromazine bound to the bulge of TAR RNA, to dock compounds from Enamine database containing 1.6 million individual compounds. Docking identified 173 compounds that were analyzed for the inhibition of HIV-1 infection. Top ten inhibitory compounds with $\mathrm{IC}_{50} \leq 6 \mu \mathrm{M}$ were selected and the three least toxic compounds, $\mathrm{T} 6780107\left(\mathrm{IC}_{50}=2.97 \mu \mathrm{M}\right), \mathrm{T} 0516-4834\left(\mathrm{IC}_{50}=0.2 \mu \mathrm{M}\right)$ and $\mathrm{T} 5628834\left(\mathrm{IC}_{50}=3.46 \mu \mathrm{M}\right)$, were further tested for HIV-1 transcription inhibition. Only T0516-4834 compound showed selective inhibition of Tat-induced HIV-1 transcription, whereas T6780107 compound inhibited equally basal and Tatinduced transcription and T5628834 compound only inhibited basal HIV-1 transcription. The T0516-4834 compound also showed strongest inhibition of HIV-1 gag RNA expression and p24 production in CEM T cells infected with HIV-1 IIIB. Of the three compounds, only the T0516-4834 compound disrupted Tat-TAR RNA interaction indicating that it might target TAR RNA. Also, of the three tested compounds, T5628834 but not T6780107 or T0516-4834 disrupted Tat-CDK9/cyclin T1 interaction. Taken together, our study identified novel compound T0516-4834 that disrupted Tat-TAR RNA interaction and inhibited Tat-induced transcription and HIV-1 infection suggesting that this compound might serve as a new lead for anti-HIV-1 therapeutics.
\end{abstract}

Keywords: HIV-1 transcription; HIV-1 Tat; TAR RNA; small molecule inhibitors

\section{Introduction}

Eradication of the Human Immunodeficiency Virus (HIV) remains a challenge despite introduction of combination anti-retroviral therapy (cART) that suppresses viral load to undetectable levels but does not eliminate viral reservoirs that remain for the life time of the host [1]. Interruption of cART regiment, which is common among HIV-1+ patients especially in resources poor countries, will result in the HIV-1 rebound and appearance of mutated viral species resistant to antiviral drugs [2]. Also, chronic long-term HIV-1 infection leads to cardiovascular and neurological diseases, metabolic syndrome, and non-infectious respiratory 
disease, which summarily contribute to HIV-1 morbidity and mortality [1-3]. As cART does not affect HIV-1 transcription, small molecules are needed to block HIV-1 transcription in order to prevent HIV-1 provirus activation and eliminate HIV-1 related chronic comorbidities. HIV-1 transcription is activated by HIV-1 Tat protein that interacts with the HIV-1 transactivation response (TAR) RNA and recruits host CDK9/cyclin T1 and other factors to induce elongation of HIV-1 transcription and facilitate full-length transcript production [6]. In the absence of Tat protein, HIV-1 transcription is terminated after the synthesis of 82 nucleotide long TAR RNA [7]. HIV-1 TAR RNA has been thought as a target for small molecule inhibitors, as it stands out as a unique HIV-1 target. Computation screening of close to 200,000 compounds identified acetylpromazine that was shown to bind the bulge of TAR RNA [4], overlapping with the binding of Tat protein that specifically interacts with the bulge [5, 6]. A more recent study utilized a virtual screening based on "scaffold-hopping" with several potential TAR RNA binding scaffolds including acetylpromazine identified molecules disrupted Tat-TAR RNA interaction with midmicromolar IC 50 [7]. Here, we utilized the existing NMR structure of TAR RNA with the bound acetylpromazine to screen in silico compounds from Enamine database. Docking identified 173 compounds that were further tested for the inhibition of HIV-1 replication in CEM cell infected with VSVg-pseudotyped HIV-1. This allowed narrowing down the candidate list to three compounds that were analyzed for their effect on Tat-induced and basal HIV-1 transcription and disruption of Tat/TAR RNA and Tat/CDK9/cyclin T1 complexes. Taken together, our study identified a novel compound that may serve as a new lead for anti-HIV-1 therapeutics.

\section{Materials and Methods}

\subsection{Materials.}

293T cells were purchased from ATCC (Manassas, VA). CEM T cells were purchased from ATCC (Manassas, VA). Protein A/G agarose beads were purchased from Sana Cruz Biotechnology (Santa Cruz, CA). Anti-cyclin T1 rabbit monoclonal antibody was purchased from Sana Cruz Biotechnology (Santa Cruz, CA). Anti-CDK9 rabbit monoclonal antibody was purchased from Sana Cruz Biotechnology (Santa Cruz, CA). Anti-FLAG mouse monoclonal antibody was purchased from Sigma (St. Louis, MO). Non-radioactive translation system was purchased from Promega. Supplemented DMEM and RPMI media were purchased from Invitrogen. Biotinylated WT TAR RNA (59 nucleotides long) and mutant delta TAR RNA (48 nucleotides long) were synthesized by Integrated DNA Technologies (Coralville, Iowa). Unless indicated, all other chemicals and enzymes were obtained from Sigma-Aldrich (St. Louis, MO, USA). Small molecules were synthesized by Enamine (Ukraine).

\subsection{Viruses and plasmids.}

HIV-1 proviral DNA, pNL4-3.Luc.R-E containing two nonsense frame shift in the Env and Vpr gene with Luciferase reporter gene cloned in place of (Nef) was obtained from NIH AIDS Reagent Program (Germantown, MD). VSVG pseudotyped HIV-1 expressing luciferase virus (HIV-1-LUCG) was generated using pNL4A3.Luc.R.E and VSVG-expressing plasmid, pHEFAVSVAG, obtained from NIH AIDS Reagent Program (Germantown, MD). Flag-Tat expressing vector was as previously described [8]. HIV-1 LTR-luciferase vector was kindly provided by Dr. Manuel LópezCabrera (Unidad de Biología Molecular, Madrid, Spain)

\subsection{Molecular docking.}

Virtual screening using docking technique of Enamine stock database was conducted with QXP program, which showed excellent results to reproduce experimental docking positions on targets with diverse binding sites. The structure of HIV-1 TAR with a small molecule (PDB ID 1LJV) was used in this study [PUBMED ID: 12079782]. The binding site was defined and, consequently, grid was generated using residues within $10 \AA$ from the bound ligand. A systematic docking 
routine sdock+ was employed to generate 300 positions per ligand and top 10 scored poses were saved. The post docking filtering was performed using multiRmsd, which allows performing hypothesis driven selection of putative complexes from QXP docking results [6].

\subsection{HIV-1 Infection.}

HIV-1-LUC-G was utilized to infect leukemic lymphoid CEM T-cells. CEM T cells were grown in RPMI medium (Invitrogen) supplemented with 10\% Fetal Bovine Serum (FBS) and 1\% antibiotic solution (penicillin and streptomycin) at $37^{\circ} \mathrm{C}$ in humidified incubator with $5 \% \mathrm{CO}_{2}$. CEM T cells were infected by HIV-1-LUC-G, cultured at $1 \times 10^{6}$ cells $/ \mathrm{mL}$ in 6 -well plates at $37^{\circ} \mathrm{C}$ and $5 \% \mathrm{CO}_{2}$ for 24 hours and then treated with different concentrations of indicated compounds.

\subsection{Luciferase Assay.}

At 48 hrs post infection, the cells were collected, washed with PBS and then incubated with 100 $\mu \mathrm{l}$ of steady light reconstituted luciferase buffer (Luclite Kit, Perkin Elmer) for 10 minutes at room temperature. Luminescence was measured using Glo-Max Microplate Multimode reader (Promega).

\subsection{Cell Proliferation Assay (MTT Assay)}

CEM T cells were seeded at concentration of $2 \times 10^{5}$ cells $/ \mathrm{mL}$ in 96 -well plates and treated with selected inhibitors at different concentrations $(1 \mu \mathrm{M}, 3 \mu \mathrm{M} 10 \mu \mathrm{M}, 30 \mu \mathrm{M}, 60 \mu \mathrm{M}$ and $100 \mu \mathrm{M})$ for 24 hrs. Next day, $10 \mu \mathrm{l}$ of MTT reagent was added to each well and incubated for 2 hours at $37^{\circ} \mathrm{C}$ in tissue culture incubator. Then $200 \mu \mathrm{l}$ of DMSO was added to each well and the absorbance at 595 $\mathrm{nm}$ was measured using a microplate reader (Bio-Rad).

\subsection{Tat-induced HIV-1 transcription based on luciferase assays}

293T cells (ATCC) were grown in DMEM media supplemented with $10 \%$ Fetal Bovine Serum (FBS) and $1 \%$ antibiotic solution (penicillin and streptomycin) at $37^{\circ} \mathrm{C}$ in humidified incubator with $5 \%$ CO2. 293T cells were seeded in 96 well plates at 30\% confluence and lipofectamine 3000 reagent (Life Technologies) was utilized for transfection. 293T cells were co-transfected with Tat-expressing vector and HIV-1 LTR- luciferase reporter gene. At $24 \mathrm{hrs}$ post transfection, inhibitors were diluted in DMEM media and added to the cells at different concentrations and incubated for another $24 \mathrm{hrs}$. At 48 hours post transfection, the cells were collected and washed with phosphate-buffered saline (PBS), and then $100 \mu \mathrm{l}$ of steady light reconstituted luciferase buffer (Lucilyte, Perkin Elmer) was added for $10 \mathrm{~min}$ at room temperature. Luminescence was measured using Glo-Max Microplate Multimode reader (Promega).

\subsection{Tat-TAR RNA interaction assay.}

Biotinylated WT TAR RNA (59 nucleotides long) and mutant delta TAR RNA with the deletions of Tat-binding bulge nucleotides 21-27 and 38-41 were synthesized by Integrated DNA Technologies (Coralville, Iowa). To prepare cell lysates containing Tat, 293T cells were cotransfected with Tat expression vector for $48 \mathrm{hrs}$ and then lysed in the Whole cell lysate buffer. To bind TAR RNA, streptavidin-agarose beads were washed with the binding buffer prepared in RNAse free water (20 mM Tris- $\mathrm{HCl} \mathrm{pH} 7.5,2.5 \mathrm{mM} \mathrm{MgCl}$, $100 \mathrm{mM} \mathrm{NaCl})$. The beads were blocked with BSA and tRNA was also added to prevent unspecific binding. Blocked beads were then incubated with $10 \mu \mathrm{g}$ of TAR RNA or $10 \mu \mathrm{g}$ of delta TAR RNA as control. Beads incubated with TAR RNA and delta TAR RNA were washed and incubated with approximately $400 \mu \mathrm{g}$ Tat lysate in presence of TAK buffer ( $50 \mathrm{mM}$ Tris-HCl, $\mathrm{pH}$ 8.0, $5 \mathrm{mM} \mathrm{MgCl}$, $5 \mathrm{mM} \mathrm{MnCl}_{2}, 10 \mu \mathrm{M} \mathrm{ZnSO}_{4}, 1$ $\mathrm{mM}$ DTT). Then $10 \mu \mathrm{M}$ of each compound was added. Beads were collected by 5 min centrifugation at $1000 \mathrm{xg}$, and the bound proteins were eluted in 1x SDS-loading buffer. Proteins then separated on 
$10 \%$ Bis-Tris gel, followed by transferring to PVDF membrane, immunoblotting with anti- Flag antibody and chemiluminescence detection as previously described [9].

\subsection{Immunoprecipitation and Western Blotting.}

293T cells transfected as described above were lysed in the whole cell lysate buffer $(50 \mathrm{mM}$ Tris-HCl, $\mathrm{pH} 7.5,0.5 \mathrm{M} \mathrm{NaCl}, 1 \% \mathrm{NP}-40,0.1 \%$ SDS) supplemented with 1x cocktail protease inhibitor (Sigma) to prevent proteolysis. Total protein concentration was determined using BCA protein assay (Bio-Rad). Lysates were stored at $-80^{\circ} \mathrm{C}$ until use. Protein A/G agarose beads (Sana Cruz Biotechnology) were blocked with 5\% Bovine Serum Albumin (BSA). Blocked beats were incubated with anti-Flag antibody then combined with protein lysate and incubated for 2 hours at $4^{\circ} \mathrm{C}$. Beads were washed with $1 \times$ TNN buffer (10 mM Tris- $\mathrm{HCl} 7.5,0.1 \% \mathrm{NP}-40$ and $\left.100 \mathrm{mM} \mathrm{NaCl}\right)$ and then heated for 5 minutes at $70^{\circ} \mathrm{C}$. Eluted proteins were loaded on $10 \%$ Bis-Tris gel and resolved in 1x MOPS running buffer supplemented with SDS and anti-oxidant (Invitrogen). The gel was blotted to PVDF membrane and overnight. The membrane was blocked in 5\% non-fat milk, washed in PBS buffer containing 0.001\% Tween and incubated with primary antibodies including Anti-CDK9 (1:4000) (Rabbit Sana Cruz, Lot No: I0414), Anti-Cyclin T1 (1:4000) (Rabbit Sana Cruz, Lot No:D1709) or anti-Flag 1:2000 (mouse) (Sigma) for 2 hrs at room temperature. The membrane was washed with PBST and incubated with HRP- conjugated anti-mouse or anti-rabbit and probed. Chemilumensence was detected with chemiluminescent substrate (Clarity ECL Western Blot Substrate Kits, Bio-Rad) using ChemiDoc XRS Imaging station (Bio-Rad).

\subsection{The p24 enzyme-linked immunosorbent assay (ELISA).}

CEM T cells were infected with fully replication competent HIV-1(IIIB). Supernatant was collected at 48 hrs post infection and p24 was measured by HIV-1 p24 antibody ELISA (PerkinElmer) using OPD as substrate. Data were analyzed using standards provided within the kit.

\subsection{Determination of HIV-1 Gag and HIV-1 reverse transcription.}

Quantitative analysis of HIV-1 RNA was conducted on total RNA isolated from CEM T cells infected with fully replication competent HIV-1(IIIB). RNA was isolated using Trizol Reagent (Invitrogen, Carlsbad, CA). For quantification of HIV-1 DNA, total DNA was extracted from 1x10 ${ }^{6}$ cells infected with HIV-1(IIIB) using total DNA isolation kit (Thermo Fischer). Real-time PCR analysis was conducted in Roche Light Cycler 480 (Roche Diagnostics) using SYBR Green Master Mix (Roche Diagnostics). PCR amplification was carried with initial preincubation for 5 min at $45^{\circ} \mathrm{C}$, then $3 \mathrm{~min}$ at $95^{\circ} \mathrm{C}$ followed by 45 cycles of denaturation at $95^{\circ} \mathrm{C}$ for $15 \mathrm{sec}$, annealing and extension at $60^{\circ} \mathrm{C}$ for $45 \mathrm{sec}$, and final extension at $72^{\circ} \mathrm{C}$ for $10 \mathrm{sec}$. Mean Cp values for Early-LTR, Late-LTR transcripts and $\beta$ - globin DNA was determined using $\Delta \Delta \mathrm{Ct}$ method. For gag quantification, total RNA (100 ng) was reverse transcribed to cDNA using Superscript RT-PCR kit (Invitrogen, Carlsbad, CA). Quantification of gag mRNA was carried using 18S RNA as a housekeeping control. Mean $\mathrm{Cp}$ values were determined and $\Delta \Delta \mathrm{Ct}$ method was used to calculate relative expression levels. The following primer sequences were used.

primers used for HIV-1 DNA and RNA quantification:

HIV gag forward: ATAATCCACCTATCCCAGTAGGAGAAAT

HIV gag reverse: TITGGTCCITGTCITATGTCCAGAATGC

Early LTR forward: GGCTAACTAGGGAACCCACTG

Early LTR reverse: CTGCTAGAGATTTTCCACACTGAC 
Late LTR forward: TGTGTGCCCGTCTGTTGTGT

Late LTR reverse: GAGTCCTGCGTCGAGAGATC

$\beta$-actin forward: CTCCCAAAGTGCTGGGATTA

$\beta$-actin reverse: CAAAGGCGAGGCTCTGTG

18SrRNA forward: CAAAGGCGAGGCTCTGTG

18SrRNA reverse: CTCCAGGTTTTGCAACCAGT

\subsection{Statistical Analysis.}

Statistical analysis was performed using GraphPad Prism 6 software (Graph Pad Software, San Diego, CA, USA). All data are presented as means with standard deviation. . Mean and Standard deviation (STDEV) were calculated for 3 repeats at each concentrations of each inhibitor. Differences between the two groups were compared using the parametric unpaired two-tailed Student's t-test. For reproducibility, experiments were repeated three time independently.

\section{Results}

\subsection{Selection of TAR RNA-binding compounds by molecular docking.}

Coordinates of HIV-1 TAR RNA model (PDBID: 1LVJ) was retrieved from PDB. The full Enamine stock database (over 2 million compounds) was pre-filtered to extract structures containing one hydrogen bond donor and one aromatic ring. This initial filtering yielded 500,136 compounds. Molecular docking results were processed to extract complexes that matched following criteria: i) a putative ligand should intercalate into RNA structure ii) form a hydrogen bond with a phosphate oxygen of U25 and iii) form stacking interactions with bases of C26, C39, U40 and, U25 (Figure 1A). The filtering was performed using docking processing suite multiRmsd software which allows performing hypothesis driven selection of putative complexes from QXP docking results. . As a result we selected 173 compounds that were screened in vitro.

\subsection{Screening small molecules library for inhibition of one round HIV-1 infection.}

To evaluate the HIV-1 inhibitory activity of TAR RNA-targeting small molecules, CEM T cells were infected with VSV-G pseudotyped pNL4-3.Luc.R-E-virus (HIV-1-LUC-G) for 24 hrs and then treated with $10 \mu \mathrm{M}$ compound. Screening of 173 compounds showed 9 compounds that inhibited HIV-1 for at least 40\% (Figure 1D). 


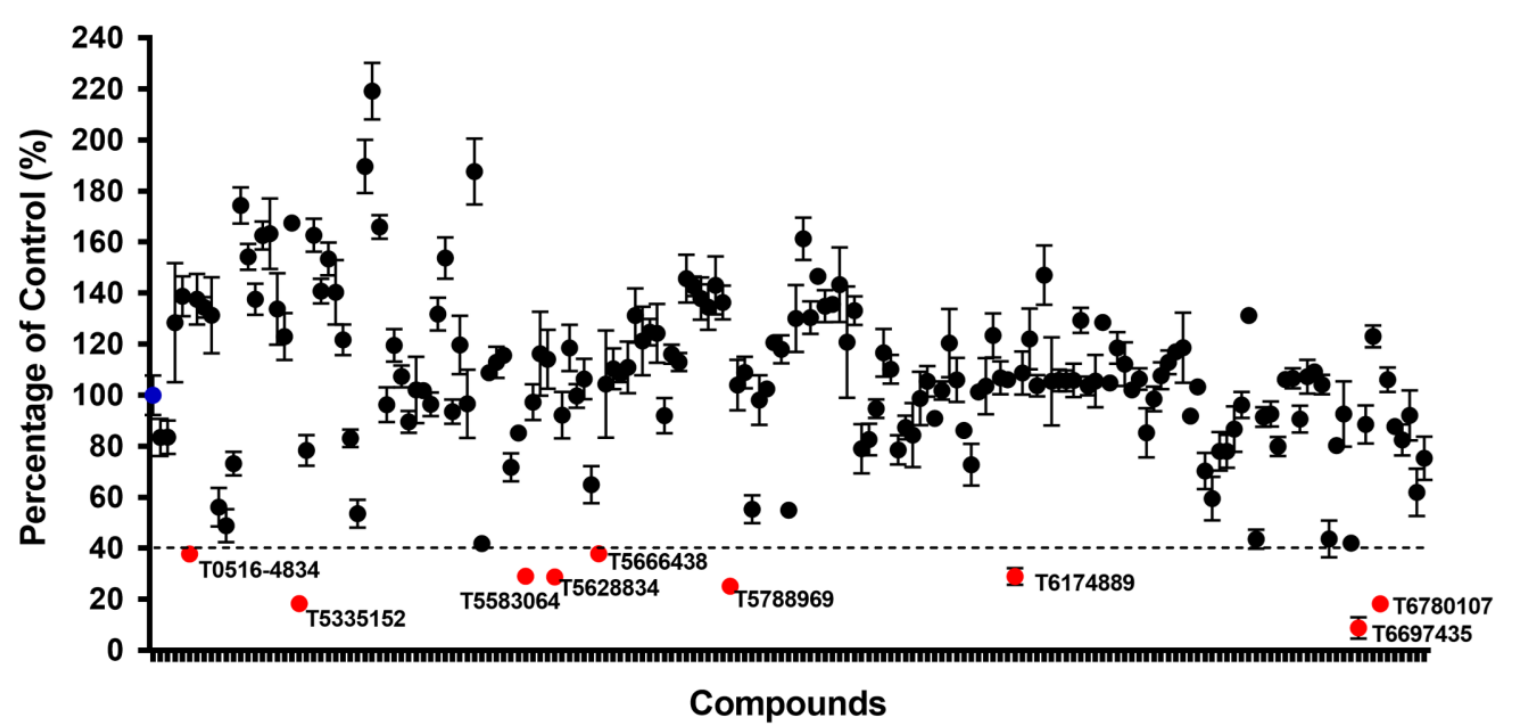

Figure 1. High throughput screening of TAR RNA-targeting small molecules for one round HIV-1-Luc infection in CEM $T$ cells. CEM T cells were seeded in 96 well plates, treated with $10 \mu \mathrm{M}$ concentration of compounds. DMSO represents $100 \%$ (blue) and percentage of inhibition was calculated for each compound. In red, compounds are shown that achieved more than $60 \%$ inhibition.

\subsection{Analysis of cytotoxicity and dose-dependent inhibition of one round HIV-1.}

Next, we determined IC50s for one round HIV-1 inhibition of the 9 identified compounds. CEM T cells were infected with HIV-1-LUC-G for $24 \mathrm{hrs}$ followed by treatment with the selected compounds for another 48 hrs. The three compounds that showed consistent HIV-1 inhibition were T0516-4834 (IC50=0.3 $\mu \mathrm{M})$, T6780107 (IC50=3.7 $\mu \mathrm{M})$, and T5628834 (IC $\left.{ }_{50}=3.7 \mu \mathrm{M}\right)$ (Figure 2A). The rest of the compounds showed either upregulation of HIV-1 infection at lower concentrations or inefficient inhibition (Figure 2B). None of the compounds showed any toxicity under $100 \mu \mathrm{M}$ concentrations (not shown). 
A

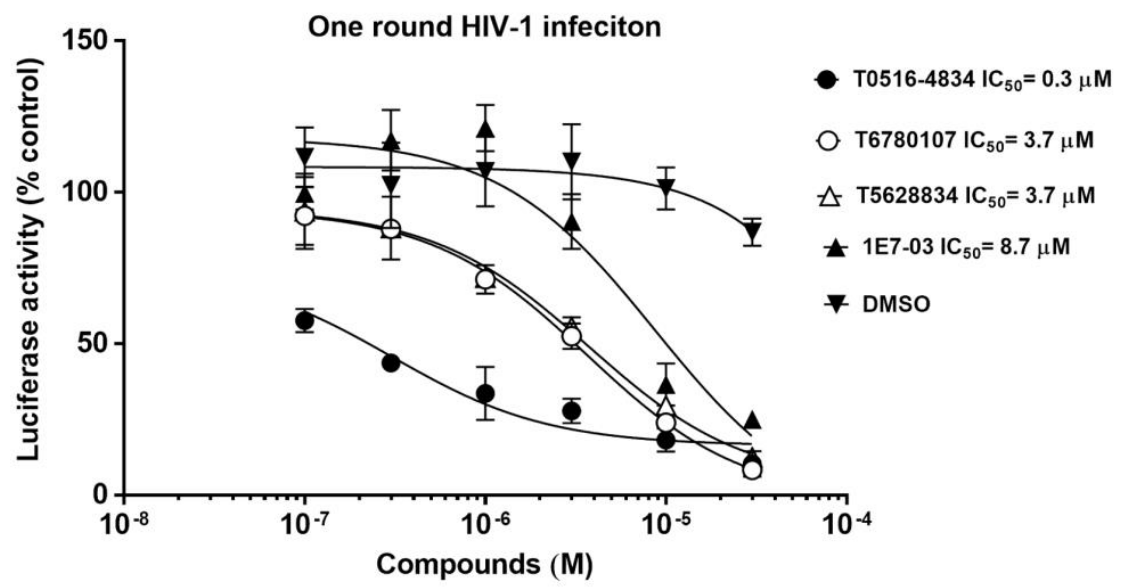

B

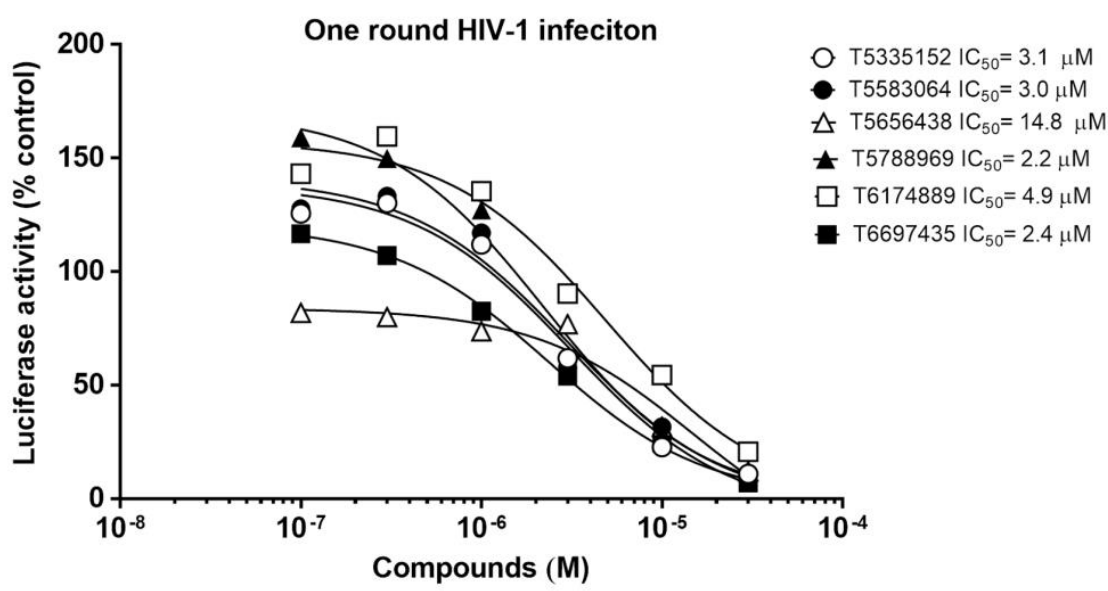

Figure 2. Dose dependent HIV-1 inhibition by top candidate compounds. CEM T cells were infected with HIV-1-LUC-G, then seeded in 96 well plate and treated with the indicated concentrations of the compounds. DMSO represents $100 \%$ and percentage of inhibition was calculated for each compound concentration.

\subsection{Effect of the selected compounds on Tat-mediated transcription and production of HIV-1 gag mRNA.}

The three selected compounds (Figure 3A) were further analyzed for their mechanism of action. To test whether the compounds interfered with HIV-1 Tat activated transcription, we utilized a luciferase reporter assay in $293 \mathrm{~T}$ cells that were transfected with a reporter plasmid expressing luciferase under the control of HIV-1 LTR and a plasmid expressing HIV-1 Tat that contained Flag tag. At 24 hrs post transfection, the cells were treated with different concentrations of the compounds for another 24 hrs. Co-expression of HIV-1 LTR reporter and Flag-Tat expressing plasmid resulted in $>40$-fold induction of transcription by Tat (Supplemental Fig.1). Dosedependent inhibition of Tat-induced transcription was observed for T0516-4834 (IC $\left.{ }_{50}=2 \mu \mathrm{M}\right)$ and $\mathrm{T} 6780107\left(\mathrm{IC}_{50}=3.5 \mu \mathrm{M}\right)$ whereas $\mathrm{T} 5628834$ showed little inhibition $\left(\mathrm{IC}_{50}=20 \mu \mathrm{M}\right)$ (Fig. 3B). Basal HIV-1 transcription was inhibited in dose-dependent manner by all three compounds with (T6780107 $\left(\mathrm{IC}_{50}=4.63 \mu \mathrm{M}\right)$ having the strongest effect followed by T5628834 $\left(\mathrm{IC}_{50}=6.2 \mu \mathrm{M}\right)$ and T05164834 ( $\left.\mathrm{IC}_{50}=15 \mu \mathrm{M}\right)$ (Fig.3C).To elucidate whether the selected compounds have an effect on HIV-1 transcription during infection with fully replication capable HIV-1, CEM T cells were infected with fully replication competent HIV-1 IIIB and gag mRNA expression was quantified by real time PCR. The strongest reduction of HIV-1 gag mRNA level was observed for T0516-4834 compound (27fold reduction) followed by T6780107 (5.5-fold reduction) and T5628834 (1.6-fold reduction, no statistical significance) (Fig. 3D). 
To further characterize the antiviral activity of the selected compounds in the context of active HIV-1 replication, we determined p24 level of in the media collected from the infected CEM T cells treated with the compounds. We observed the strongest reduction of p24 in the T0516-4834 treated cells (4-fold reduction) followed by T6780107 (2.2-fold reduction) and T5628834 (1.26-fold reduction) (Fig.3E). This observation paralleled the effect of these compounds on gag mRNA levels in HIV-1 infected cells and suggested that T0516-4834 was the best inhibitor.

A

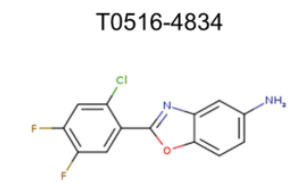

T6780107

T5628834
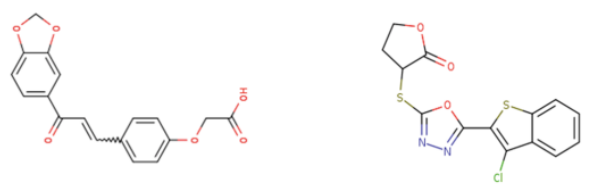

B

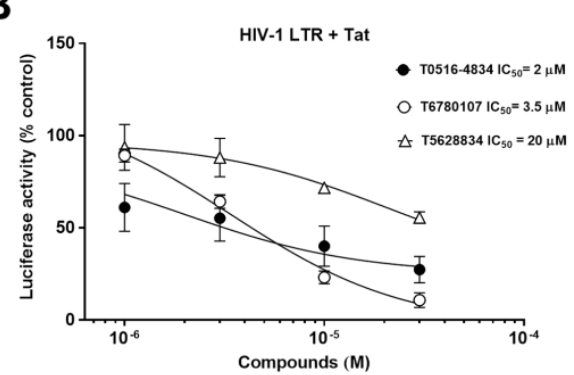

C

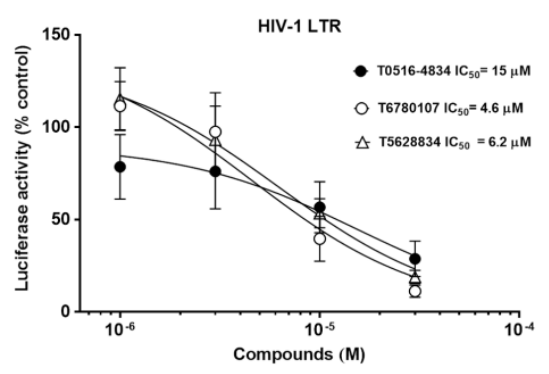

\section{D}

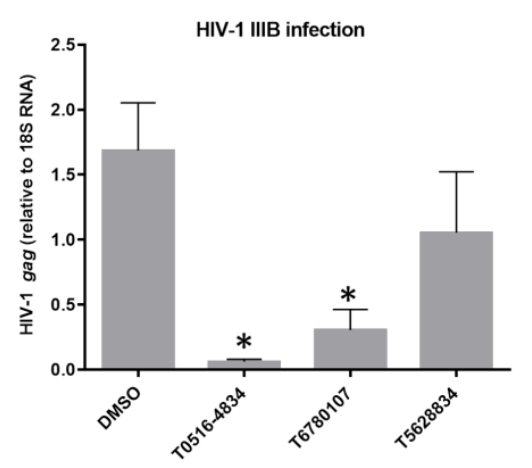

E

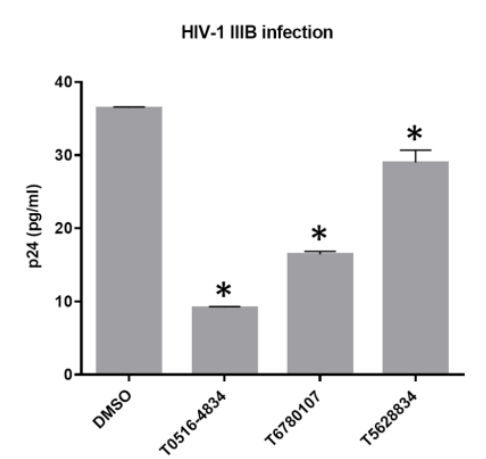

Figure 3. Inhibition of Tat-medicated transcription and HIV-1 replication by TAR RNA-targeting compounds. A, shown are structures for T0516-4834, T6780107 and T5628834 compounds. B and C, inhibition of HIV-1 Tat activated and basal HIV-1 transcription. 293T cells were transfected with HIV-1 LTR reporter and Tat expressing vector (panel B) or only HIV-1 LTR reporter (panel C) and treated with indicated concentrations of the compounds for 24 hrs. Luciferase activity was measured as described in Materials and Methods. Data were plotted in Prims 6. D and E, inhibition of HIV-1 replication. CEM T cells were infected with HIV-1 IIIB for 48 hrs followed by RNA isolation and real-time PCR analysis for HIV-1 gag mRNA )panel D) or p24 ELISA (panel E) as described in Materials and Methods. Asterisks indicate $\mathrm{p}<0.01$ in comparison to the DMSO controls.

\subsection{Disruption of Tat TAR interaction by T0516-4834.}

As we were targeting TAR RNA, we next tested whether the selected compounds had an effect on the interaction between Tat and TAR RNA. Biotinylated 58 nt TAR RNA was immobilized on avidin-containing agarose beads and used to pull down Tat from lysates of 293T cells that were transfected with Flag-Tat expressing vector. The lysates were supplemented with $10 \mu \mathrm{M}$ compounds (Fig.4). We observed Tat binding to the immobilized TAR RNA (Fig.4A, lane 2) but not the beads alone (Fig.4A, lane 4) or to the beads-immobilized TAR with the deletion of the bulge (Fig.4A, lane 4, delta). We observed significant disruption of Tat binding to TAR RNA with T05164834 compound (2.5-fold inhibition, $\mathrm{p}=0.023$ ) (Fig 4A, lane 8 and Fig. 4B) In contrast, two other compounds showed little or no effect with no statistical significance on Tat-TAR RNA binding. 
A

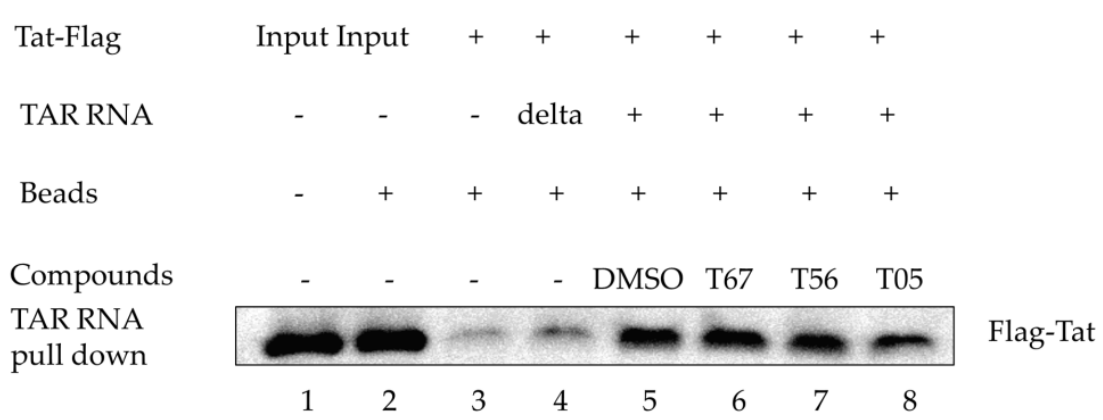

B

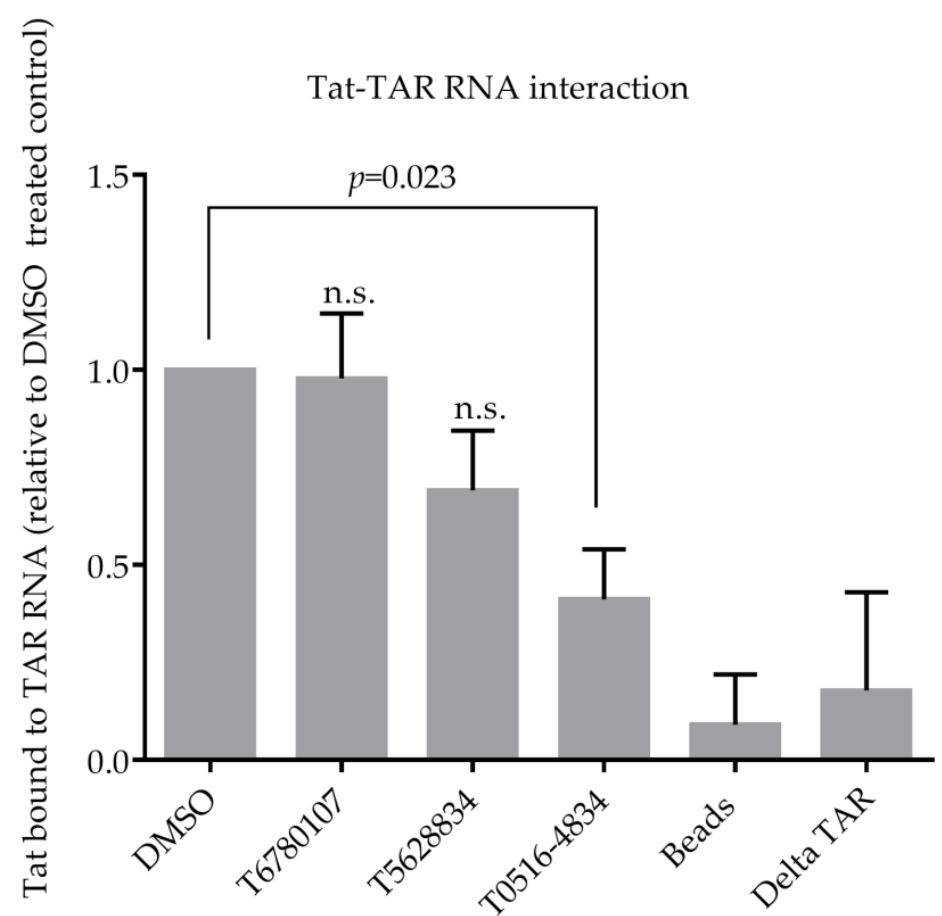

Figure 4. Disruption of Tat TAR-RNA interaction by T0516-4834 compound. A, Binding between Tat and TAR RNA was performed using protein lysate prepared from transfection 293T cells with Tat-Flag plasmid for 48 hrs. Beads were blocked with BSA and tRNA then incubated with TAR RNA followed by precipitation with Tat lysate and treatment with $10 \mu \mathrm{M}$ of each compound. Tat protein was eluted with SDS loading buffer, resolved on SDS-PAGE and detected with anti- Flag antibody. B, Quantification of Tat protein bound to TAR RNA. Results from two independent experiments were plotted using Prism 6.

\subsection{Effect of the compounds on Tat/CDK9/Cyclin T1 complex formation.}

As only one compound disrupted Tat-TAR RNA interaction, we extended our analysis to evaluate whether other inhibitory compounds might disrupt the interaction of Tat with CDK9/cyclin T1 complex and, thus, inhibited HIV-1 transcription. We utilized immunoprecipitation assays using ells lysate collected from 293T cells co-transfected with Flag-Tat for $24 \mathrm{hrs}$, followed by treatment with the $20 \mu \mathrm{M}$ compounds for another $24 \mathrm{hrs}$. Immunoprecipitations were carried out with protein A/G agarose beads that were pre-blocked with BSA and then incubated with cell lysates. Anti-Flag antibody was used to precipitate Flag-tagged Tat and the Tat-associated proteins that were eluted from the beads with SDS-PAGE loading buffer resolved on SDS-PAGE and detected with different antibodies. Tat protein expression levels were slightly increased in the cells treated with any compounds (Fig.5A). Among the tested compounds, 
T5628834 had the strongest effect (4-fold reduction) on CDK9 level associated with Tat (Fig.5B). Compound T0516-4834 showed moderate reduction (1.4 -fold reduction) and compound T6780107 had no effect (Fig.5B). Cyclin T1 protein levels associated with Tat were reduced by T5628834 compound (1.6 -fold reduction) whereas T6780107 and T0516-4834 compounds had no significant effects (Fig. 5C).

A
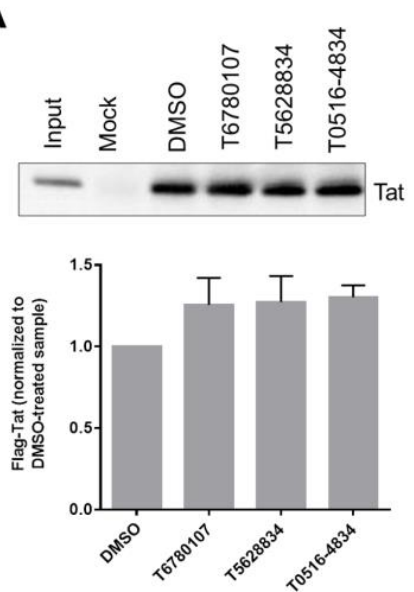

B
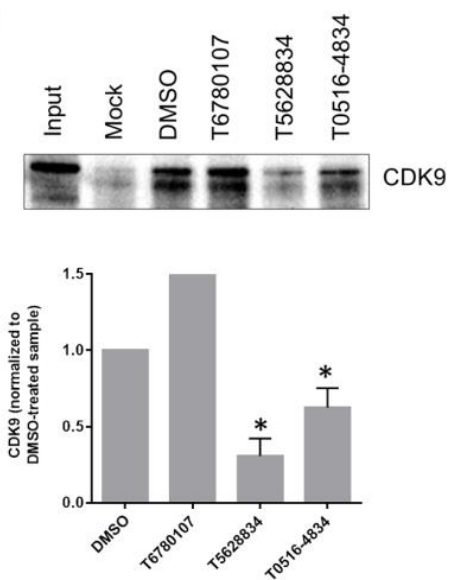

C
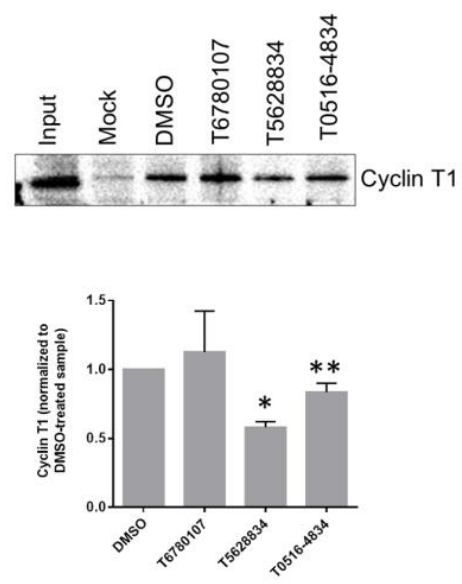

Figure 5. Effect of TAR RNA targeting compounds on Tat interaction with CDK9/Cyclin T1. Tat precipitated from $293 \mathrm{~T}$ cells co-transfected with Flag-Tat and, where indicated, treated with $20 \mu \mathrm{M}$ compounds. Protein levels were determined by Western blotting. A, Tat expression determined by antiFlag antibodies. B, CDK9 protein expression determined using anti CDK9 antibodies. C, Level of cyclin T1 determined by anti-cyclin T1 antibodies. Quantification was performed using Prism 6 from three independent experiments. ${ }^{*}$ indicates $\mathrm{p}<0.01$ and ${ }^{* *}$ indicates $\mathrm{p}<0.05$ in comparison to the DMSO controls.

\subsection{Effect of the selected compounds on HIV-1 reverse transcription.}

We next analyzed the effect of the compounds on early steps of HIV-1 infection. CEM T cells were infected with HIV-1 IIIB. HIV-1 DNA was isolated at 48 hrs post infection and quantified using primers for early and late RT products. Azitotymidine (AZT) used as positive control reduced HIV-1 early LTR (Supplemental Fig. 2A, 1.8-fold reduction) and late LTR (Supplemental Fig.2B, 63-fold reduction). No significant reduction was observed for either of the tested compounds compared to AZT (Supplemental Fig. 2A-B).

\subsection{Model of TAR RNA binding for T0516-4834.}

To visualize T0516-4834 compound interaction with TAR RNA, we built in silico model that shows binding of the discovered hit to HIV-1 TAR RNA bulge formed by U23, U25, C39, G26,C39 and U40 (Fig. 6A). The top performing compound benzoxazole T0516-4834 binds into the crevice formed by these nucleotides forming pi-pi staking interaction with $\mathrm{U} 25$ and $\mathrm{U} 40$ and its chlorine atom forms van der Waals contacts with the cleft formed by C39 and U40 (Fig.6A). T0516-4834 compound showed similar binding to compound 2 [10] which interacts with TAR RNA within the same cleft (Fig. 6B). 
A

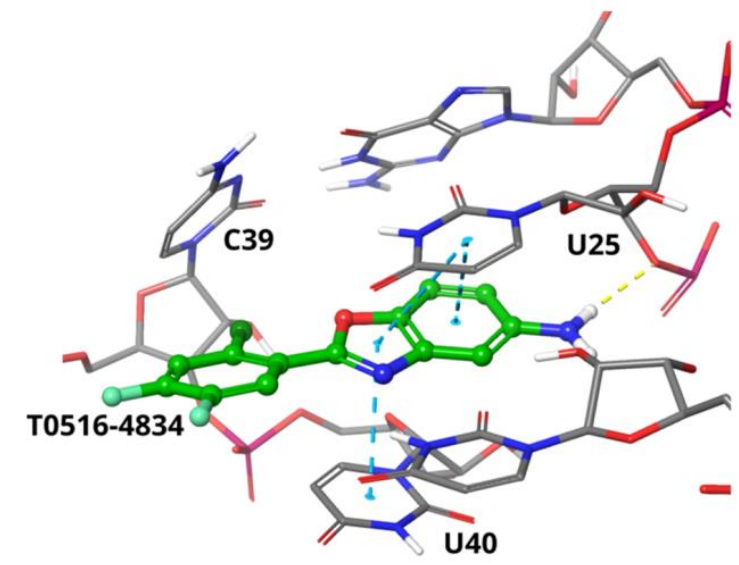

B

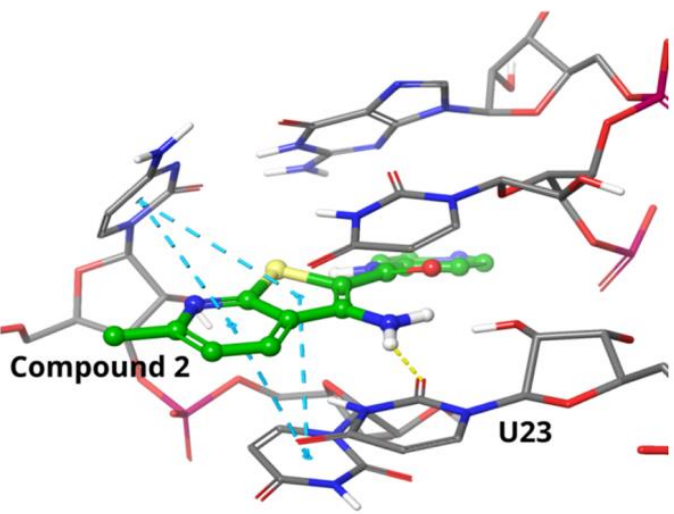

Figure 6. Binding models of complexes of HIV-1 TAR RNA with T0516-4834 (panel A ) and a compound 2 [10] (panel B). Nucleotides that form the crevice are shown in sticks. Pi-pi stacking is shown as light blue dashed lines.

Taken together, we identified novel molecule that prevented Tat interaction with TAR RNA and efficiently inhibited Tat-induced HIV-1 transcription and viral replication.

\section{Discussion}

In this study, we screened over 1.6 million individual compounds from Enamine database against TAR RNA structure and identified 173 compounds that were further analyzed to identify the most efficient inhibitor and to gain inside on its mechanism of action. Our top candidate, T05164834 compound showed selective inhibition of Tat-induced HIV-1 transcription, efficient inhibition of HIV-1 gag mRNA expression and p24 production and disrupted Tat-TAR RNA interaction. A recent study has reported discovery and optimization of thienopyrimidines as HIV-1 TAR RNA binding molecules [10]. These TAR RNA binding small molecules displaced Tat-derived peptide from TAR RNA $40 \mu \mathrm{M}$ IC50 and demonstrated non-canonical TAR RNA binding as determine by NMR [10]. Our top performing compound benzoxazole T0516-4834 bound in silico in to TAR RNA into the crevice formed by U25 and U40 nucleotides, similar to the previously identified compound 2 [10] (Fig. 6). Thienopiridines inhibited HIV-1-induced cytopatic effect at 28 M EC50, without cytotoxicity [11]. In our study, we observed HIV-1 inhibition with $0.3 \mu \mathrm{M}$ IC 50 in one round HIV-1 infection. Thus our compound describe here is superior despite close structure similarity. In addition to thienopyrimidines, several additional classes of molecules were shown to bind to TAR RNA including a cyclic peptide mimic of Tat [12], amilorides [13], a tricationic oligopyridylamide, ADH-19 [14]. However, none of these compounds were able to achieve submicromolar or even low micromolar HIV-1 inhibition.

To understand whether the identified compounds selectively inhibited Tat-induced transcription, we analyzed their effect on Tat-induced transcription and compared to inhibition of basal HIV-1 transcription. All three tested compounds exhibited a dose-dependent reduction in Tatmediated transcription activation, and also showed does-dependent inhibition HIV-1 basal transcription. Only T0516-4834 compound showed stronger inhibition for Tat-mediated transcription compared to basal transcription suggesting that this compound had selectivity toward the inhibition of Tat-induced transcription. Previously reported TR87 compound, which binds with high affinity to TAR RNA, reduces Tat-mediated transcription and down-regulates HIV-1 
replication through the disturbance of Tat TAR RNA interaction [23] Similarly, CGP64222, which was shown to bind Tat protein and to induce a conformational change in TAR RNA bulge, blocks HIV-1 replication and specifically inhibits Tat-transactivation [24]. As out top compound T05164834 compound prevented Tat-TAR RNA interaction suggests that its mechanism of action includes disruption of this key interaction. The most promising HIV-1 transcription inhibitor, didehydrocortistatin A (DCA), targets Tat protein by binding to the positively charged lysine of Tat's TAR RNA binding domain and decreases the binding affinity of Tat to TAR RNA [25]. Analysis of the effect of the three tested compounds on Tat binding to CDK9/cyclin T1 showed that T5628834 and to a lesser extent T0516-4834 compound reduced the this binding. Thus T5628834 compound might disrupt binding of Tat to Cyclin T1. The mechanism of T6780107 compound that down-regulated HIV-1 replication and transcription, remains to be defined.

The tested compounds inhibited HIV-1 transcription and had no effect on HIV-1 reverse transcription. Thus, the early steps of HIV-1 infection including, entry, binding and reverse transcription are not likely to be affected by the compounds. Utility of HIV-1 transcription inhibitors is highlighted by our recent study in which activation of HIV-1 in macrophages was the major contributing factor to lung inflammation in HIV-1 transgenic (HIV-Tg) mice [15, 16]. Treatment of HIV-Tg mice with 1E7-03, an HIV-1 transcription inhibitor ameliorated LPS-induced lung inflammation and prevented mice death [16]. As individuals living with HIV-1 have higher prevalence of non-infectious lung disease [17], future testing of the compounds described here are warranted in HIV-1 related pathologies including lung inflammation. Because cART therapy has no effect on HIV transcription, development of transcriptional inhibitors such as T0516-4834 or 1E\&-03 are needed and might help to treat HIV-1 -related chronic complications.

\section{Conclusions}

Our present study successfully identified T0516-4834 compounds that downregulated HIV-1 transcription and replication and disrupted Tat binding to TAR RNA. Moreover, T0516-4834 was found to be selective for Tat-induced transcription. Our study confirms that disruption of Tat-TAR RNA interaction is a feasible approach for inhibition of to inhibit HIV-1 transcription and replication. The identified compounds might serve as new leads for anti-HIV-1 therapeutics.

Author Contributions: conceptualization S.N and D.K.; methodology, S.N.; formal analysis, A.A., N.K and S.N.; investigation, A.A., A.I., N.K. , X.L., and S.W.; resources, D.K. and S.N.; data curation, S.N.; writing-original draft preparation, S.N.; writing-review and editing, A.A., D.K. and S.N.; supervision, A.I, N.K. and S.N.; visualization, D.K.; funding acquisition, S.N.

Funding: This research was funded by NIH Research Grants (1P50HL118006, 1R01HL125005, 5U54MD007597, and 1UM1AI26617), and the District of Columbia Developmental Center for AIDS Research grant (P30AI087714). The content is solely the responsibility of the authors and does not necessarily represent the official views of the National Institutes of Health.

Acknowledgments: We thank NIH AIDS Research and Reference Reagent Program for pHEFVSVG expression vector (courtesy of Dr. Lung-Ji Chang) and pNL4-3.Luc.R-E- (Courtesy of Dr. Nathaniel Landau). We also thank Dr. Manuel López- Cabrera (Unidad de Biología Molecular, Madrid, Spain) for the gift of HIV-1 LTR-luciferase expression vector.

Conflicts of Interest: The authors declare no conflict of interest.

\section{References}


1. Friis-Moller, N.; Sabin, C. A.; Weber, R.; d'Arminio Monforte, A.; El-Sadr, W. M.; Reiss, P.; Thiebaut, R.; Morfeldt, L.; De Wit, S.; Pradier, C.; Calvo, G.; Law, M. G.; Kirk, O.; Phillips, A. N.; Lundgren, J. D.; Data Collection on Adverse Events of Anti, H. I. V. D. S. G., Combination antiretroviral therapy and the risk of myocardial infarction. The New England journal of medicine 2003, 349, (21), 1993-2003.

2. Wand, H.; Calmy, A.; Carey, D. L.; Samaras, K.; Carr, A.; Law, M. G.; Cooper, D. A.; Emery, S.; Committee, I. T. I. C., Metabolic syndrome, cardiovascular disease and type 2 diabetes mellitus after initiation of antiretroviral therapy in HIV infection. Aids 2007, 21, (18), 2445-53.

3. Calligaro, G. L.; Gray, D. M., Lung function abnormalities in HIV-infected adults and children. Respirology 2015, 20, (1), 24-32.

4. Du, Z.; Lind, K. E.; James, T. L., Structure of TAR RNA complexed with a Tat-TAR interaction nanomolar inhibitor that was identified by computational screening. Chemistry $\mathcal{E}$ biology 2002, 9, (6), 707-12.

5. Delling, U.; Roy, S.; Sumner-Smith, M.; Barnett, R.; Reid, L.; Rosen, C. A.; Sonenberg, N., The number of positively charged amino acids in the basic domain of Tat is critical for trans-activation and complex formation with TAR RNA. Proceedings of the National Academy of Sciences of the United States of America 1991, 88, (14), 6234-8.

6. Roy, S.; Delling, U.; Chen, C. H.; Rosen, C. A.; Sonenberg, N., A bulge structure in HIV-1 TAR RNA is required for Tat binding and Tat-mediated trans-activation. Genes Dev 1990, 4, (8), 1365-73.

7. Renner, S.; Ludwig, V.; Boden, O.; Scheffer, U.; Gobel, M.; Schneider, G., New inhibitors of the TatTAR RNA interaction found with a "fuzzy" pharmacophore model. Chembiochem : a European journal of chemical biology 2005, 6, (6), 1119-25.

8. Ammosova, T.; Berro, R.; Jerebtsova, M.; Jackson, A.; Charles, S.; Klase, Z.; Southerland, W.; Gordeuk, V. R.; Kashanchi, F.; Nekhai, S., Phosphorylation of HIV-1 Tat by CDK2 in HIV-1 transcription. Retrovirology 2006, 3, 78.

9. Ivanov, A.; Lin, X.; Ammosova, T.; Ilatovskiy, A. V.; Kumari, N.; Lassiter, H.; Afangbedji, N.; Niu, X.; Petukhov, M. G.; Nekhai, S., HIV-1 Tat phosphorylation on Ser-16 residue modulates HIV-1 transcription. Retrovirology 2018, 15, (1), 39.

10. Abulwerdi, F. A.; Shortridge, M. D.; Sztuba-Solinska, J.; Wilson, R.; Le Grice, S. F.; Varani, G.; Schneekloth, J. S., Jr., Development of Small Molecules with a Noncanonical Binding Mode to HIV-1 Trans Activation Response (TAR) RNA. Journal of medicinal chemistry 2016, 59, (24), 11148-11160.

11. Sztuba-Solinska, J.; Shenoy, S. R.; Gareiss, P.; Krumpe, L. R.; Le Grice, S. F.; O'Keefe, B. R.; Schneekloth, J. S., Jr., Identification of biologically active, HIV TAR RNA-binding small molecules using small molecule microarrays. Journal of the American Chemical Society 2014, 136, (23), 8402-10.

12. Bardaro, M. F., Jr.; Shajani, Z.; Patora-Komisarska, K.; Robinson, J. A.; Varani, G., How binding of small molecule and peptide ligands to HIV-1 TAR alters the RNA motional landscape. Nucleic Acids Res 2009, 37, (5), 1529-40.

13. Patwardhan, N. N.; Ganser, L. R.; Kapral, G. J.; Eubanks, C. S.; Lee, J.; Sathyamoorthy, B.; Al-Hashimi, H. M.; Hargrove, A. E., Amiloride as a new RNA-binding scaffold with activity against HIV-1 TAR. MedChem Comm 2017, 8, (5), 1022-1036.

14. Maity, D.; Kumar, S.; Curreli, F.; Debnath, A. K.; Hamilton, A. D., alpha-Helix-Mimetic Foldamers for Targeting HIV-1 TAR RNA. Chemistry 2019, 25, (30), 7265-7269.

15. Jerebtsova, M.; Ahmad, A.; Kumari, N.; Rutagarama, O.; Nekhai, S., Macrophage HIV-1 Gene Expression and Delay Resolution of Inflammation in HIV-Tg Mice. Viruses 2020, 12, (3).

16. Jerebtsova, M.; Ahmad, A.; Niu, X.; Rutagarama, O.; Nekhai, S., HIV-1 Transcription Inhibitor 1E7-03 Restores LPS-Induced Alteration of Lung Leukocytes' Infiltration Dynamics and Resolves Inflammation in HIV Transgenic Mice. Viruses 2020, 12, (2).

17. Morris, A.; Crothers, K.; Beck, J. M.; Huang, L.; American Thoracic Society Committee on, H. I. V. P. D., An official ATS workshop report: Emerging issues and current controversies in HIV-associated pulmonary diseases. Proceedings of the American Thoracic Society 2011, 8, (1), 17-26.

(C) 2021 by the authors. Submitted for possible open access publication under the terms and conditions of the Creative Commons Attribution (CC BY) license (http://creativecommons.org/licenses/by/4.0/). 